\title{
Beyond exemplars and prototypes as memory representations of natural concepts: A clustering approach is
}

\author{
Timothy Verbeemen ${ }^{\mathrm{a}}$, Wolf Vanpaemel ${ }^{\mathrm{a}}$, Sven Pattyn ${ }^{\mathrm{a}}$, \\ Gert Storms $^{\mathrm{a}, *}$, Tom Verguts ${ }^{\mathrm{b}}$ \\ a Department of Psychology, University of Leuven, Tiensestraat 102, 3000 Leuven, Belgium \\ ${ }^{\mathrm{b}}$ Ghent University, Ghent, Belgium
}

Received 27 February 2006; revision received 15 September 2006

Available online 13 November 2006

\begin{abstract}
Categorization in well-known natural concepts is studied using a special version of the Varying Abstraction Framework (Vanpaemel, W., \& Storms, G. (2006). A varying abstraction framework for categorization. Manuscript submitted for publication; Vanpaemel, W., Storms, G., \& Ons, B. (2005). A varying abstraction model for categorization. In B. Bara, L. Barsalou, \& M. Bucciarelli (Eds.), Proceedings of the 27th annual conference of the Cognitive Science Society (pp. 2277-2282). Mahwah, NJ: Lawrence Erlbaum). This framework assumes a continuum between highly abstract memory representations (prototypes) and highly detailed representations of concept members (exemplars). Comparison stimuli for categorization are obtained by taking for each category the centroids of a set of clusters, produced by $K$-means clustering, effectively producing the Generalized Context Model (GCM; Nosofsky, R. M. (1986) Attention, similarity, and the identification-categorization relationship. Journal of Experimental Psychology: General. 115, 39-57) and the Single-Prototype Model as extreme cases. The clustering version of the Varying Abstraction Framework was fit on a set of novel, to-be-classified fruits and vegetables (Smits, Storms, Rosseel, \& De Boeck, 2002) and on a new set of novel, to-be-classified carnivores and herbivores. Better fit values were clearly obtained for a model based on intermediately abstract representations, indicating a strategy where people compare the novel stimuli to a set of multiple prototypes. This sheds a new light on the prototype versus exemplar discussion that has dominated the literature over the past 25 years.
\end{abstract}

(c) 2006 Elsevier Inc. All rights reserved.

Keywords: Varying abstraction; Prototypes; Exemplar models; Semantic concepts; Categorization

\footnotetext{
The first author is a research assistant of the Fund for Scientific Research, Flanders. This project was in part sponsored by Grant OT/05/27 of the University of Leuven research council to Gert Storms. The first two authors contributed equally to this manuscript. We thank Lance Rips and Edward Wisniewski for their comments and Rudi D'Hooge for his help in gathering and describing the stimuli for Study 2 .

* Corresponding author. Fax: +3216325 916.

E-mail addresses: timothy.verbeemen@psy.kuleuven.be
} (T. Verbeemen), gert.storms@psy.kuleuven.be (G. Storms).
One of the most fundamental abilities in cognitive functioning is the capacity to classify stimuli into a limited number of categories. These categories are used to organize and structure objects in the world around us. Categorization makes the world more predictable, because many unknown properties of newly encountered stimuli can be induced with sufficient certainty as soon as the stimulus is recognized as a member of a certain category (Estes, 1994). 
The question of how categories are stored in memory and how they are activated in category related decisions has intrigued psychologists for decades. In what has been called the classical view on categories (Komatsu, 1992), it was assumed that categories are well-defined. More specifically, it was assumed that a category was represented in memory by a set of singly necessary and jointly sufficient characteristics. The categorization process was understood to consist of a simple check of the applicability of the defining feature set. In the seventies, a large amount of empirical evidence was presented that contradicted this classical view (for overviews, see Murphy, 2002; Smith \& Medin, 1981) and, except for historical reasons, the view disappeared from the literature.

In the last three decades, the literature on concepts and categories has been dominated by two rather different traditions. (For overviews of the literature in this domain, see, e.g., Komatsu, 1992; Medin \& Coley, 1998; Murphy, 2002; Smith \& Medin, 1981.) The first of the two traditions studies lexicalized semantic concepts (like fruits, furniture, apple, and chair). The central research questions in this tradition are how such concepts are represented in semantic memory and which processes operate on these representations (e.g., Hampton, 1979, 1993; Rogers \& McClelland, 2004; Rosch \& Mervis, 1975; Storms, De Boeck, \& Ruts, 2000, 2001). Because the natural language concepts studied in this tradition refer to existing things in the world, and because these existing concepts often cannot easily be manipulated, many of these studies are correlational (e.g., Hampton, 1979; McRae, Cree, Westmacott, \& de Sa, 1999; but see Rips, 1989; Ross \& Murphy, 1999). Many studies within the domain of natural language have proposed a prototype view on semantic concept representation (e.g., Hampton, 1993; Lakoff, 1987; Rosch, 1978). In this view, a concept is assumed to be represented as an abstract summary representation, consisting of features that are characteristic, rather than strictly defining for the concept. The summary representation is assumed to be built up through abstraction over previously encountered concept members. Categorization of a newly encountered entity is assumed to be based on a comparison of the entity with such prototypes, with a decision in favor of the most similar prototype representation.

Studies in the second tradition use category learning experiments in laboratory settings. The stimuli used in category learning experiments are artificial and can be optimally controlled. Typically, a limited set of training stimuli belonging to two competing categories has to be classified and feedback is given after every training trial. Afterwards, a set of transfer stimuli, not previously presented, has to be classified without feedback. Through careful design of the underlying feature structures of the categories, predictions of competing storage and retrieval models can be optimally distinguished and eval- uated (e.g., Lamberts, 2000; Medin \& Schaffer, 1978; Nosofsky, 1986; Verguts, Ameel, \& Storms, 2004; Verguts, Storms, \& Tuerlinckx, 2003). In this research tradition, an exemplar view of categories has been dominant. The models developed in this domain assume that categories are represented as memory traces of encountered exemplars and that no abstraction across these stored exemplars takes place. Categorization is assumed to be based on the similarity of a newly encountered entity towards all stored exemplars of the competing categories. Evidence for these models comes from categorization experiments designed to compare the predictions of exemplar and prototype models. In the majority of these studies (e.g., Medin \& Schaffer, 1978; Medin, Altom, \& Murphy, 1984; Nosofsky, 1992), different versions of the exemplar model outperformed the prototype model (but see Smith \& Minda, 2000, 2002 for nuances to this general conclusion, and Nosofsky \& Johansen, 2000 for a reply).

The experimental approach led to the development of formal versions of categorization models that can be rigorously tested and compared. However, the drawback of this gain of control in laboratory experiments is the associated loss in external validity. Several authors have questioned the relevance of these artificial category learning experiments for the study of natural language concepts (e.g., Malt \& Smith, 1984; Markman \& Ross, 2003; Murphy, 2003). In particular, in light of the fact that each situation we encounter is completely new, it is not clear how to understand the notion of an 'exemplar' in the context of natural language concepts like fruits or birds (Storms, 2004). At a theoretical level, different versions of the exemplar view have been presented, depending on the assumptions made about the number and nature of the stored instances, about the presence or absence of forgetting, and so on (Barsalou, 1990). At one extreme, exemplar representations may involve no abstraction at all. In this version, representations consist only of specific memory traces of particular previously encountered instances (e.g., Reed, 1972). At the other extreme, an exemplar representation might be a family resemblance representation that abstracts across different specific instances (Komatsu, 1992). An intermediate position was taken by Rosch (1975) who assumed that only the most typical exemplars are activated as category representation in memory.

To phrase the problem more concretely, is a robin an exemplar of the category bird? Or is a European robin (Erithacus rubecula) an exemplar? Or is the specific European robin that I saw through the window this morning an exemplar? And when I see that same bird tonight flying by, is that the same or another memory trace that counts as an exemplar? It is intuitively hard to believe that people activate all exemplars of, for instance, humans, every time they categorize something as a human being. We can safely assume that a typical 
adult living in an urbanized area has encountered thousands of exemplars of human beings, if not millions, when counting different experiences involving the same person as different exemplars. In a nutshell, situations we encounter are hierarchically organized at different levels, and it is not clear which level should be designated as the exemplar level.

The problem can even be extended to the stimuli used in artificial category learning experiments. In this context, exemplars are usually equated with stimuli that were presented during the experimental procedure. Determining the appropriate level of abstraction comes down to the question whether frequently presented exemplars count as a single or as multiple exemplars. Nosofsky (1988) provided evidence that exemplars do not refer to actual objects, but rather to the encounters with these objects. A similar position has been defended by Barsalou, Huttenlocher, and Lamberts (1998). However, one can question whether a theory which assumes no abstraction at all has ever been tested in any of the artificial categorization studies. In designing experiments, researchers assume that the representation of the stimuli is based on the dimensions that they manipulate (e.g., color, form, size, and position of the stimulus). However, if participants do not abstract these dimensions from other information that may in principle be stored in memory (e.g., the trial number or uncontrolled variations in illumination across trials), they might not be able to learn the categories. In this sense, each training exemplar is a sort of a prototype consisting of a set of abstracted features that, in principle, can apply also to other exemplars that differ on some other, irrelevant features.

In previous attempts to apply the exemplar model in the context of natural language concepts, exemplar levels were defined pragmatically as 'a hierarchical level below the level of the studied concepts' (Elvevåg, Storms, Heit, \& Goldberg, 2005; Smits et al., 2002; Storms et al., 2000, Storms, De Boeck, \& Ruts, 2001). Heit and Barsalou's (1996) instantiation principle, for instance, states that people instantiate a semantic concept like mammals with hierarchically lower concepts such as $\operatorname{dog} s$, horses, etc. However, the empirical evidence they gathered cannot determine how far down the instantiation process goes. More specifically, when judging the typicality of mammal with respect to animal, it is possible that participants retrieve one (or more) instantiation(s) defined at the level of dog or horse, evaluate their typicality as animals, and then judge the typicality of mammals within the category animals based on the typicality of its instantiations. But it is also possible that they further instantiate $d o g$ with (one or more) particular kind(s) of dogs, like dobermann, or even with specific memorized exemplars of dobermanns (as is assumed by many exemplar models). Thus, the evidence gathered thus far in favor of instantiation processes is not conclusive about the exact level of abstraction of the activated memory representations and therefore does not yield direct evidence for activation of specific memory traces of encountered exemplars.

The hierarchical organization of semantic knowledge does not only hold difficulties for the exemplar view, it also affects the tenability of the prototype view. Evidence for the prototype view in the study of natural language concepts has mostly concentrated on so-called superordinate and basic level concepts (i.e., categories like furniture and chair, respectively; see Rosch, Mervis, Gray, Johnson, \& Boyes-Braem, 1976). As concepts are situated higher up in the hierarchical structure (i.e., as they become more abstract), they are more heterogeneous (i.e., the diversity of their exemplar set increases). As a consequence, abstraction into a single summary representation for such hierarchically higher concepts is more difficult. Intuitively, it is easy to think of an average granny smith apple, discarding the idiosyncratic peculiarities of all the individual granny smith apples that we ever encountered. But looking at a higher category level, it is not at all obvious what the abstract summary representation of fruits (i.e., the collection of diverse things like apples, litchis, kiwis, bananas, and so on) looks like, let alone what summary representations of concepts like food consist of.

The fuzzy status of exemplars and prototypes has not escaped categorization theorists. Most solutions to the problem are inspired by or at least related to the concept of unsupervised competitive learning developed in neural network theory (e.g., Love, Medin, \& Gureckis, 2004; Rumelhart \& Zipser, 1985; Schyns, 1991; Verguts et al., 2004). Neglecting all model specifics that these authors have incorporated, unsupervised competitive learning can essentially be described as follows (e.g., Herz, Krogh, \& Palmer, 1991). Suppose the input space is two-dimensional. Each input node codes for one particular dimension (so there are also two input nodes; see Fig. 1a). Input nodes are initially randomly connected to a set of output nodes. Each time an input pattern is presented, all output units are activated depending on the match between the input pattern vector and the weight vector attached to the output unit (both of which are, in this example, two-dimensional). Then, a competition occurs in which only the most strongly activated output unit remains active (in variations of this scheme, such as a Kohonen network, neighboring units of the winning node also remain slightly active). This winner of the competition is allowed to change its weights in the direction of the input pattern, so that if the same input pattern is presented on a later trial, it will win with even higher probability. The result of this process is that the output units will function as clusters: each output unit will be located in a portion of the input space that is strongly represented in the training set (see Fig. 1b). For this reason, unsupervised competitive learning is 
a

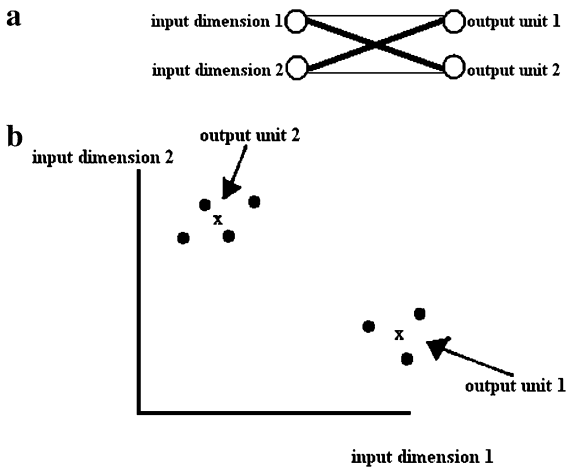

Fig. 1. Graphical overview of unsupervised competitive learning. (a) Network topology for the example described in the text. Note that the input dimensionality (2) is the same as the dimensionality of the weight vector attached to an output unit. (b) Representation of the result of the learning process. Each dot represents one (of 7) stimuli presented in the experiment. Each $x$ represents (the weight vector attached to) an output unit. For example, output unit 1 has a strong connection to input dimension 1 but a weak connection to input dimension 2 (see (a)).

formally related to, and the outcome in some cases identical to, $K$-means clustering (Hastie, Tibshirani, \& Friedman, 2001).

The indeterminate status of exemplars and prototypes can now be stated more precisely. An unsupervised learning model with the same number of output nodes as there are stimuli, will probably end up as an exemplar model: each output unit will cover one of the stimuli in the input space. In the graphical representation of Fig. 1b, there will be seven $x$ 's, and each of the $x$ 's will cover one of the dots. On the other hand, an unsupervised learning model with only one output unit (for each category) will end up as a prototype model, and its input weight configuration will be placed about midway between all the stimuli in Fig. $1 \mathrm{~b}$.

All of this suggests that the classical dichotomy between the exemplar and the prototype view should be extended toward a more general view in which the prototype and the exemplar model are extreme poles of a continuum. The aim of the present paper is, first, to present a formal framework in which exemplar models, prototypes models, and anything in between can be described in a uniform way. The second aim is to evaluate this framework on two empirical datasets and determine the level of abstraction that is used when categorization decisions have to be made. Our approach has as its starting point the Varying Abstraction Framework, which is an extension of the Generalized Context Model (GCM); (Nosofsky, 1984), recently proposed by Vanpaemel, Storms, and Ons (2005). The Varying Abstraction Framework is a generic framework in which a stimulus set that constitutes a particular category is partitioned into a number of subsets, each represented by a summary representation that abstracts over the exemplars belonging to that subset.

One possibility would be to apply an unsupervised learning procedure on the input space to determine the partitioning of the stimulus set of every candidate category (e.g., the example of Fig. 1b would lead to a partition of two subsets, containing 3 and 4 stimuli, respectively). However, it is not our aim to model the learning process in detail. Therefore, and because of the formal similarity between the outcome of unsupervised learning and $K$-means clustering, we will use the latter as a convenient approximation to the result of the learning process. The resulting approach is called the $K$-means Varying Abstraction Framework. Note that the exemplar and the prototype model are special cases of the $K$-means Varying Abstraction Framework.

In the next section, the framework and its instantiations (exemplar model, prototype model, $K$-means Varying Abstraction Framework) will be described in a more formal manner. Afterwards, the framework will be applied to categorization data from two experiments with natural language concepts.

\section{The Varying Abstraction Framework}

The Varying Abstraction Framework is based on the key idea that intermediate abstraction processes may underly category representations and categorization decisions. Other models that incorporate the same key idea but that start from different theoretical assumptions have been presented (e.g., the Rational Model of Anderson, 1991 the SUSTAIN model of Love et al., 2004 and the Mixture Model of Rosseel, 2002). The Varying Abstraction Framework that we applied in the present study elaborates on Nosofsky's $(1984,1986)$ generalized context model (GCM). (The relation between our approach and the models of Anderson, Love et al., and Rosseel will be spelled out more in detail in the General Discussion section.) In the present section, we start with an outline of Nosofsky's GCM. Next, we present the Varying Abstraction Framework, and finally we introduce the $K$-means Varying Abstraction Framework.

\section{A generic framework}

In the GCM (Nosofsky, 1984, 1986), categorization is a function of similarity towards all relevant stored exemplars. The model was formulated as a generalization of Medin and Schaffer's (1978) context model to incorporate stimuli that differ on continuous characteristics rather than binary dimensions. The GCM fitting procedure starts from a psychological space that is obtained with a multidimensional scaling procedure 
(MDS; see, e.g., Takane, Young, \& De Leeuw, 1977) on proximity measures of all stimulus pairs involved. The coordinates of these stimuli are then used as input for the model. This implies that a choice is made as to the orientation of the axes representing this solution. The reason is that distances between stimuli in a space do not alter as a result of rotating the coordinate axes. Following common practise in the scaling literature, the coordinate axes of the multidimensional scaling solution were fixed according to a principal axes rotation. In this procedure, the first axis is chosen so that this dimension captures most of the variation of the multidimensional stimulus distribution. Likewise, the second axis is chosen in such a way that it again captures as much of the remaining variation as possible, and so on. This provides a plausible approach to uncovering a good and sparse representation of the data structure (see, e.g., Lee, 2001).

When the underlying psychological space is determined, the predicted categorization probabilities can be calculated. In the case of two categories, A and B, the probability that stimulus $x$ is classified in category $\mathrm{A}$ is given by:

$\mathrm{P}(\mathrm{A} \mid \mathrm{x})=\frac{\beta_{\mathrm{A}} \eta_{x \mathrm{~A}}}{\beta_{\mathrm{A}} \eta_{x \mathrm{~A}}+\left(1-\beta_{\mathrm{A}}\right) \eta_{x \mathrm{~B}}}$,

where $\beta_{\mathrm{A}}$ varies between 0 and 1 and serves as a response bias parameter towards category A. The quantities $\eta_{x \mathrm{~A}}$ and $\eta_{x \mathrm{~B}}$ denote the similarity of stimulus $x$ toward category A and B, respectively. In the GCM, this similarity is equal to the similarity of stimulus $x$ to all stored exemplars of category A and B, respectively, which is formalized as:

$\eta_{x \mathrm{~A}}=\sum_{j \in \mathrm{A}} \exp -\left[c\left(\sum_{d=1}^{D} w_{d}\left|y_{x d}-y_{j d}\right|^{r}\right)^{1 / r}\right]^{q}$.

The symbol $D$ denotes the number of dimensions. Further, $y_{x d}$ and $y_{j d}$ are the coordinates of stimulus $x$ and exemplar $j$ of category A (or B for $\eta_{x \mathrm{~B}}$, respectively) on dimension $d$. The weight of the $d$ th dimension is denoted by $w_{d}$. The sum of the weights is restricted to sum to 1 . The power metric, determined by the value of $r$, is usually given a value of either 1 or 2, corresponding to city-block and Euclidean distance, respectively. The sensitivity parameter $c$ determines the overall scaling of the distances. The parameter $q$ determines the shape of the similarity gradient, where typically the values 1 or 2 are used, corresponding to an exponential or a Gaussian shape, respectively. We will refer to this model as the Full-Exemplar Model.

Prototype models based on the GCM have also been developed. Much of the traditional research that was based on artificial stimuli used a very limited set of training stimuli that varied on a set of binary dimensions (or features). Because averaging over discrete features is not always sensible, the prototype was usually conceived as an ideal example of a category with modal values for that category. The MDS used in the GCM allows for a more straightforward way of defining a prototype. In particular, the object created by taking, on each dimension, the average coordinate over all members of the category, is a straightforward definition of a prototype. The similarity of stimulus $x$ toward category A is now conceptualized as the similarity of stimulus $x$ toward the prototype of category $\mathrm{A}$, denoted as $\mathrm{P}$ :

$\eta_{x \mathrm{~A}}=\exp -\left[c\left(\sum_{d=1}^{D} w_{d}\left|y_{x d}-y_{P d}\right|^{r}\right)^{1 / r}\right]^{q}$,

where $y_{P d}$ denotes the coordinate of the prototype of category $\mathrm{A}$ on dimension $d$. This coordinate is calculated as the mean value of all stored members of category A. We will refer to (3) in combination with (1) as the SinglePrototype Model.

\section{The Varying Abstraction Framework}

The Full-Exemplar and the Single-Prototype Models can be considered to be two extreme views. Between these two extreme positions regarding abstraction (i.e., the Single-Prototype and Full-Exemplar Model), there is a whole spectrum of intermediate abstractions. The idea of the Varying Abstraction Framework (Vanpaemel \& Storms, 2006, Vanpaemel et al., 2005) is to first make a partition ${ }^{1}$ of the category exemplars for each category involved and then to construct, for every subset of exemplars, a prototype by averaging over all the exemplars in that subset. These prototypes are called the pseudo-exemplars and are used to represent the category. When a category is partitioned in $K$ different sets, there are $K$ different pseudo-exemplars. The set of these pseudo-exemplars is denoted as $E$. The similarity of stimulus $x$ toward category $\mathrm{A}$ is now

$\eta_{x \mathrm{~A}}=\sum_{k \in E} \exp -\left[c\left(\sum_{d=1}^{D} w_{d}\left|y_{x d}-y_{k d}\right|^{r}\right)^{1 / r}\right]^{q}$,

where $y_{k d}$ denotes the coordinate of a pseudo-exemplar $k$ of category A on dimension $d$. This coordinate is calculated as the mean value of all members of the subset on that dimension. (Note that expression (4) is very similar to expression (2), the only difference being that the sum is taken over the $K$ different sets instead of $n_{\mathrm{A}}$ exemplars.) Defining a set of pseudo-exemplars for every category suffices to define a model. Therefore, we will refer to such a model as a pseudo-exemplar model. In that sense, the Varying Abstraction Framework is a family of pseudo-exemplar models.

\footnotetext{
${ }^{1}$ A partition of a set $S$ is a collection of disjoint, non-empty subsets of $S$ whose union is $S$.
} 
Since every partition defines a set of pseudo-exemplars, a combination of partitions (one partition for each category involved) picks out one specific pseudo-exemplar model. The Varying Abstraction Framework incorporates the Single-Prototype and Full-Exemplar Models when $K=1$ and $K=N$ for each category, respectively. In the first case, the partition consists of only one subset, containing all stored category members. There is only one pseudo-exemplar, which equals the category prototype. In the second case, each subset contains exactly one exemplar, and hence the pseudo-exemplars correspond to the original exemplars. All intermediate levels are incorporated in the model also. Importantly, except for the two extreme levels, there are different pseudoexemplar models at each level of abstraction. As an example, with three stimuli a, b, and c, there is one partition at level $K=1$ ( $\{\mathrm{a} \mathrm{b} \mathrm{c}\})$, three partitions at level $K=2(\{\{\mathrm{a}\},\{\mathrm{b}, \mathrm{c}\}\},\{\{\mathrm{b}\},\{\mathrm{a}, \mathrm{c}\}\},\{\{\mathrm{c}\},\{\mathrm{a}, \mathrm{b}\}\})$, and one partition at level $K=3(\{\mathrm{a}\},\{\mathrm{b}\},\{\mathrm{c}\})$. Applying the Varying Abstraction Framework allows one to determine which partition gives the best fit to the data, instead of merely comparing the two most extreme cases.

\section{The K-means Varying Abstraction Framework}

The number of different partitions of a set of $N$ elements is given by its Bell number, denoted as $\operatorname{Bell}(N)$. (The example of the previous paragraph shows that Bell $(3)=5$.) Hence, when there are two possible categories $\mathrm{A}$ and B with $N_{\mathrm{A}}$ and $N_{\mathrm{B}}$ exemplars, respectively, the Varying Abstraction Model generates $\operatorname{Bell}\left(N_{\mathrm{A}}\right) \times$ $\operatorname{Bell}\left(N_{\mathrm{B}}\right)$ models. The Bell number rises quite steeply as a function of $N$. When all categories are small, it is computationally feasible to explore all possible pseudo-exemplar models. Vanpaemel, Pattyn, and Storms (2006), for instance, analyzed thirty data sets based on the 5-4 category structure (Medin \& Schaffer, 1978). In this structure there are two categories, one with five, and one with four exemplars. In that case, the Varying Abstraction Framework defines $52 \times 15$ models and it is possible to consider them all. However, in natural language, the categories are usually much larger. In such cases, the general Varying Abstraction Framework cannot be fully applied anymore in a reasonable amount of time. For example, when there are two categories with thirty exemplars each, the Varying Abstraction Framework generates more than $10^{47}$ models.

A plausible way to reduce the number of partitions and hence the number of compared models follows from the assumption that partitions are guided by an unsupervised process, as explained above. Instead of considering all possible partitions at every level of abstraction $K$, one can restrict oneself to only a single partition for every level $K$ by taking the psychologically most plausible grouping of the stimuli, merging exemplars based on similarity of the stimuli. Not only should very similar stimuli be allowed to merge into a single summary representation, but very dissimilar stimuli should be kept separate as reference objects for the to-be-classified items. This goal is obtained using $K$-means clustering (Hastie et al., 2001), a data-analytic technique that seeks to optimally partition the exemplar set in a predefined number of $K$ subsets. Hence, at each level of abstraction there is now only one partition left, leading to only $N$ partitions for a category with $N$ elements, which is a drastic reduction relative to the original number $\operatorname{Bell}(N)$. By letting $K$ vary from 1 to $N$ (with $N$ the number of stored category exemplars), and with two rival categories $\mathrm{A}$ and $\mathrm{B}$, the number of models to be compared reduces to $N_{\mathrm{A}} \times N_{B}$. We will refer to this version of the Varying Abstraction Framework as the $K$-means Varying Abstraction Framework. As such, $K$-means clustering is implemented here to serve as an unsupervised constraint on people's representational accuracy.

\section{Study 1: categorization of fruits and vegetables}

In this section, we will present an application of the $K$-means Varying Abstraction Framework to a natural language data set consisting of the two superordinate categories fruits and vegetables taken from Smits et al. (2002). The choice of a set of natural language concepts defined at the superordinate level allows a good test of the framework because the exemplars here are basic level concepts. Importantly, fruits and vegetables can be considered each other's contrast categories (Verbeemen, Vanoverberghe, Storms, \& Ruts, 2001) and the vast majority of naturally growing foods can be classified into one of these two categories. Furthermore, instantiations of these categories are basic level concepts like oranges, spinach, etc. that can, in principle, be treated as separately stored exemplars. Alternatively, they can be grouped in subsets (e.g., citrus fruits, leave vegetables, or other groups that may be difficult to lexicalize), or they can be grouped at the level of the studied categories (i.e., fruits and vegetables). Moreover, it seems reasonable to assume that representations of exemplars of these basic level categories (e.g., of specific red cabbages, specific passion fruits, etc.), if they exist at all, will be highly similar and their (minor) differences will not affect categorization decisions much.

Smits et al. (2002) analyzed a stimulus set consisting of pictures of 79 well-known items for the categories fruits and vegetables, and 30 novel and mostly exotic foods that were completely unknown to participants. The set of well-known fruits and vegetables was taken from Storms, De Boeck, Van Mechelen, and Ruts (1996), where 25 participants each generated 10 exemplars for the respective categories. Items with a generation frequency of 2 or higher were retained. The names 
of both the well-known and novel stimuli are given in Appendix A. ${ }^{2}$

In the Storms et al. (1996) study, 30 participants generated features for the two categories (fruits and vegetables) and the 10 most frequently generated features for both categories were selected. Since three features were generated for each of the two studied categories, 17 features were retained. Smits et al. (2002) then instructed 10 participants to complete a feature applicability rating task for all stimuli (i.e., 35 fruits, 44 vegetables, and 30 novel stimuli) and the 17 selected features. ${ }^{3}$ In this task, subjects had to indicate whether a feature applied to a (pictorially presented) item by filling in 1 or 0 . Summing over the individual responses then produced a feature applicability vector for each item with values between 0 and 10 . A similarity matrix was then obtained by correlating the feature applicability vectors for all 109 stimuli. A different group of participants classified the pictures of the well-known and the novel stimuli as belonging to either fruits or vegetables. The well-known fruits and vegetables were categorized as fruits in $90 \%$ and $6 \%$ of the cases, respectively. For the novel foods, $47 \%$ of fruit classifications were made, with proportions for individual items varying from 0 to $100 \%$. Details about the observed proportions for both well-known and novel stimuli are given in Appendix A.

In order to obtain a geometric representation of the stimulus set, the derived similarities between the 109 old and novel fruits and vegetables were analyzed with ALSCAL (Takane et al., 1977). A three-dimensional ordinal solution with principal-axis rotation was chosen that explained approximately 96 percent of the variance. The resulting coordinates were then used as input for the models to be tested.

Smits et al. (2002) then predicted the categorization of the novel foods in the categories fruits and vegetables based on the geometric versions of the Full-Exemplar Model (i.e., the GCM) and the Single-Prototype Model and found a clear advantage for the Full-Exemplar Model. This may be interpreted as an indication that subjects, when incorporating novel members into wellknown concepts, do not refer to single abstract representations. Given the heterogeneity of large concepts such as fruits and vegetables, however, a test for intermediate

\footnotetext{
${ }^{2}$ It is important to notice that in Dutch (or in biology, for that matter), there is no "correct" classification of fruits or vegetables. As a result, these concepts depend highly on consensus between speakers. Because the Dutch word for a plant's fruit ("vrucht") and the category fruit ("fruit") are not the same, there may be large differences with English speakers' intuitions. For this reason, the novel items cannot be divided into fruits or vegetables according to a criterion that makes sense in Dutch.

${ }^{3}$ Taking the most frequently generated features ensures that the analysis is not obscured by potentially unreliable features that are important to only a few participants.
}

representations as an explanatory basis for categorization seems warranted.

\section{Fitting the K-means Varying Abstraction Framework}

In the illustration presented here we fitted the different models of the $K$-means Varying Abstraction Framework to the categorization data of the 30 novel stimuli. The first step was to apply $K$-means clustering to each category separately in order to find the cluster centers, and hence the pseudo-exemplar coordinates. The wellknown items were considered the stored items, as they were generated from memory by participants in an exemplar generation study. This means that there were 35 stored exemplars in the category fruits and 44 stored exemplars in the category vegetables. Every cluster configuration, ranging from 1 cluster to 35 and 44 clusters for the two concepts, respectively, was calculated. Hence, for each of the two categories, clustering was applied to the well-known stimuli based on the three dimensions as produced by ALSCAL.

The coordinates from the $K$-means clustering were used as the coordinates of the $K$ pseudo-exemplars for each category. In total, there were $35 \times 44=1540$ models, corresponding to the different combinations of the abstraction levels for each of the two studied concepts. All these models were fitted to the categorization data by maximum likelihood estimation.

Since all pseudo-exemplar models have the same numbers of parameters, we can compare the different pseudo-exemplar models using $-2 \ln (L)$, where $L$ is the likelihood value evaluated at the best fitting parameter values. Lower values of $-2 \ln (L)$ correspond to better model fits. When two models have the same number of parameters, the difference in $-2 \ln (L)$ between the models (i.e., $-2 \ln \left(L_{\text {MODEL1 } 1}\right)$ and $\left.\left(-2 \ln \left(L_{\text {MODEL2 }}\right)\right)\right)$ is a rough approximation to $2 \ln \left(B_{12}\right)$, where $B_{12}$ is called the Bayes Factor of Model 1 as compared to Model 2. Under the assumption that both models are a priori equally likely, it measures the evidence provided by the data in favor of Model 1 as opposed to Model 2. A value of $2 \ln \left(B_{12}\right)$ that exceeds 10 is interpreted as very strong evidence against Model 2. For an extensive discussion of these issues, see Kass and Raftery (1995, pp. 777-778).

\section{Results and discussion}

We will only discuss models fitted with an exponential decay function $(q=1)$ and Euclidean distances $(r=2)$ as this resulted in clearly better fit values than other values of $q$ and $r$. All other parameters of the $K$-means Varying Abstraction Framework are estimated (bias for fruit $\beta_{\mathrm{A}}$; sensitivity $c$; and the dimensional weights $w_{D}$ which sum to 1 ). The results are summarized in Figs. 2a and b. Fig. 2a shows a a three-dimensional 
view of the fit of the different models of the framework, with better fitting models corresponding to lower values on the vertical axis. The same information is shown in Fig. 2b, a two-dimensional plot in which the number of clusters for the two categories is displayed in grid form and the fit of the models is indicated with gray values: darker shades correspond to worse fitting models and lighter shades correspond to better fitting models.

Table 1 gives an overview of the fits and resulting parameter values of the Single-Prototype, The FullExemplar Model and the best fitting pseudo-exemplar model. The results showed a better fit for the Full-Exem- plar Model as compared to the Single-Prototype Model. However, the actual optimum was not situated at the Full-Exemplar Model. The pseudo-exemplar model with the best fit had 24 clusters for fruits and 14 clusters for vegetables. It had a $-2 \ln (L)$ value of 137.90 . The differences between the Single-Prototype Model and the best fitting model, and the Full-Exemplar Model and the best fitting model $(167.97-137.90=30.07$ and $149.22-$ $137.90=11.32$, respectively) are large enough to reject both the Single-Prototype Model and the Full-Exemplar Model as the best-fitting models. Importantly, as can be seen from Figs. $2 a$ and $b$, there was a relatively smooth

$\mathbf{a}$

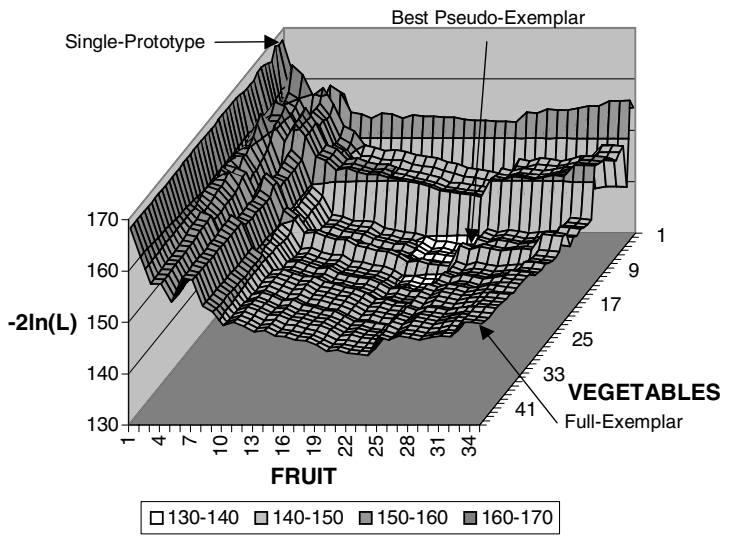

b

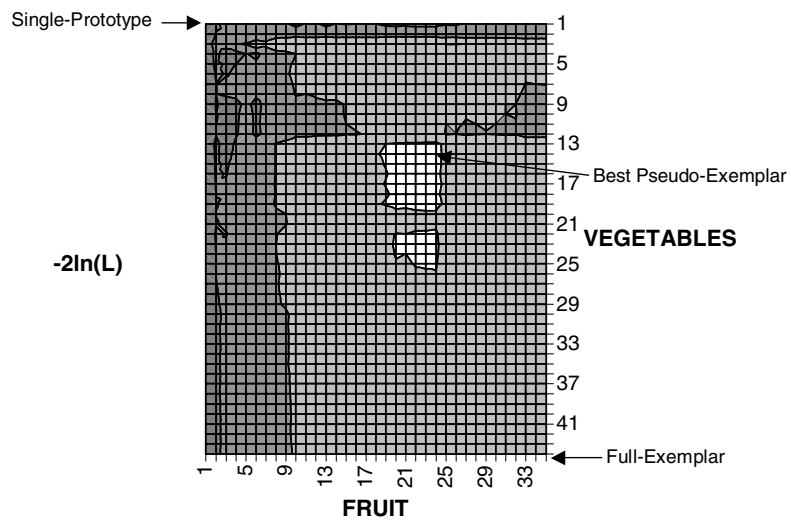

Fig. 2. $-2 \ln (L)$-values for all models for the concepts fruits and vegetables as seen on a surface plot. The number of clusters for each category is indicated on the axis, (a) gives a view from the side, (b) from the top. Orientations are identical. The optimal, SinglePrototype, and Full-Exemplar model are indicated.

Table 1

Fit value $(-2 \ln (L))$, explained variance $\left(R^{2}\right)$, sensitivity $(c)$, bias $\left(\beta_{\mathrm{A}}\right)$ for the category fruit, and dimension weights for the SinglePrototype, Full-Exemplar, and best fitting pseudo-exemplar model in Study 1

\begin{tabular}{lccccccc}
\hline Model & $-2 \ln (L)$ & $R^{2}$ & $c$ & $\beta_{\mathrm{A}}$ & $w_{1}$ & $w_{2}$ & $w_{3}$ \\
\hline Single-prototype & 167.97 & .78 & 0.806 & 0.341 & 0.986 & 0.000 & 0.013 \\
Full-exemplar & 149.22 & .85 & 4.472 & 0.313 & 0.151 & 0.056 & 0.793 \\
Best pseudo-exemplar & 137.90 & .87 & 5.349 & 0.214 & 0.158 & 0.058 & 0.785 \\
\hline
\end{tabular}


decrease towards this minimum, indicating that the optimum was not just due to spurious factors. We can therefore safely conclude that the true optimal value was situated at least somewhere around this minimum. Thus, the categorization performance of the participants in the fruits and vegetables task seems more likely to be explained by activation of representations at an intermediate level of abstraction. In other words, the results suggested that participants used representations that are more abstract than individual exemplars of the studied concepts defined at the basic level, but that are more detailed than single prototypes.

There was an asymmetry concerning the number of pseudo-exemplars that were required for the category fruits (24 clusters) and the category vegetables (14 clusters). To reach the optimal categorization prediction, fruits required more pseudo-exemplars than vegetables even though it has less stored basic level exemplars to be compared with. This is further illustrated by the actual cluster assignments as produced by $K$-means clustering. In the case of vegetables, there were always less singletons (i.e., clusters consisting of just one single element) in any of the cluster levels (except of course when $K=N$ ). In the case of fruits, many of the clusters are singletons, with for instance rhubarb as a single cluster from the start on (i.e., even when fruits was divided in only two clusters). In the optimal partition, 18 of the 24 clusters for fruits consisted of singletons. For vegetables, only 5 of the 14 clusters consisted of a single exemplar. Furthermore, most of the singletons of fruits (like meddler and pomegranate) were highly atypical and very different from most other fruits. It therefore seems sensible not to group them together with any other fruits. In the case of vegetables, there are simply less singletons.

Figs. $2 \mathrm{a}$ and $\mathrm{b}$ further show that not only the model with the best fit, but also other intermediate abstraction models with good fit values, are defined by more fruit than vegetable clusters. This finding strengthens our confidence in the conclusion that the categorization decisions caused the activation of more fruit representations than vegetable representations and thus that our knowledge of fruits is represented in a more detailed way than our knowledge of vegetables.

Most of the clusters in the best fitting pseudo-exemplar model, shown in Table 2, seem intuitively acceptable, either based on the appearance of the food items or on their being used together in the kitchen in certain preparations. However, there are a few odd clusters. In the best fitting cluster representation for fruits, the exemplars papaya and fig were grouped together in one cluster, just as star fruit and melon, and grapefruit and dates were. In the case of vegetables, pumpkin and windownasty formed a cluster, just as endives, artichoke and beans. The fact that these clusters were produced implies that they must be close together in the three-dimensional
Table 2

Clusters produced for the concepts fruits and vegetables in the best fitting pseudo-exemplar model

\begin{tabular}{ll}
\hline Fruits & \\
Cluster 1 & Meddler \\
Cluster 2 & Rhubarb \\
Cluster 3 & Blackberry, raspberry, strawberry \\
Cluster 4 & Peanuts \\
Cluster 5 & Chestnut \\
Cluster 6 & Nectarine, orange, tangerine, plum, cherries \\
Cluster 7 & Pomegranate \\
Cluster 8 & Banana \\
Cluster 9 & Cactus fruit \\
Cluster 10 & Fig, papaya \\
Cluster 11 & Litchi, mango, passion fruit \\
Cluster 12 & Pineapple \\
Cluster 13 & Coconut \\
Cluster 14 & Kiwi \\
Cluster 15 & Ground-nut \\
Cluster 16 & Grapes \\
Cluster 17 & Apple \\
Cluster 18 & Avocado \\
Cluster 19 & Lime \\
Cluster 20 & Pear \\
Cluster 21 & Walnut \\
Cluster 22 & Lemon \\
Cluster 23 & Star fruit, melon \\
Cluster 24 & Grapefruit, date \\
Vegetables & \\
Cluster 1 & Lentils \\
Cluster 2 & Chick peas, Brussels sprouts, \\
& broccoli, peas, chervil \\
Cluster 12 & Sea weed \\
Cluster 14 & Window-nasty, pumpkin \\
\hline Cluster 4 & Princess broad bean, zucchini, haricots \\
Cluster 5 & Pemato \\
Cluster 6 & Egg plant \\
Cluster 7 & Artichoke, beans, endives \\
Cluster 8 & Parsley, lettuce, spinach \\
Cluster 9 & Cucumber, paprika \\
Cluster 10 & Chicory, red cabbage, cauliflower, white cabbage, \\
& carrots, garlic, turnip, radish, mushroom, onion, \\
& Celery, pipe onion, leek, celeriac, fennel, \\
& \\
&
\end{tabular}

solution provided by Smits et al. (2002), but it is not obvious why. One possibility is that people simply grouped together similar exemplars when solving the task. Indeed, the $K$-means clustering procedure is intended to reflect the result of an unsupervised learning process, but we make no assumptions as to when exactly this learning process took place. Possibly, unsupervised learning (clustering) took place during the categorization experiment in the sense that people grouped together similar-looking stimuli. 
Another possible explanation resides in the similarity data, rather than in the solution that we obtained. Indeed, a potential problem was that Smits et al.'s similarity matrix was based on only 17 features and these features were generated for the studied concepts: People were asked to give features for the superordinates fruit and vegetables, not their basiclevel exemplars. This may have resulted in a too limited set of features (such as, e.g., "is edible"). As a consequence, some exemplars may have had unjustified high calculated similarities only because none of the features really discriminated between them. Selecting features generated for the category exemplars may solve this problem, because the generated feature set will be larger and better suited to differentiate the basic level exemplars. The second study discussed in this paper was designed to have a similarity matrix based on sufficiently discriminating features to counter this problem.

\section{Study 2: categorization of carnivores and herbivores}

Study 2 was designed to replicate the main finding from Study 1. More specifically, we wanted to find out whether intermediate levels of abstraction outperform the extreme levels of abstraction for another pair of well-known natural language concepts that are defined at the superordinate level. A second goal was to obtain a more detailed underlying similarity representation than the one used in Study 1. Therefore, we used features generated for the concept exemplars that were defined at the basic level (Rosch et al., 1976), instead of features generated for the superordinate concepts.

\section{Methods}

\section{Stimuli}

For the replication of the findings from Study 1, another pair of superordinate level categories was needed that fulfilled the following criteria (Storms et al., 2001). First, the two categories can be considered each other's contrast categories and exhaust more or less an overarching semantic domain. Second, there must exist a sufficiently large set of stimuli that are novel to the participants but that can be categorized in one of the two categories. Third, in order to apply a similar paradigm as in Study 1, it should be possible to obtain a similarity representation that includes, besides the novel stimuli, a set of well-known members of both categories that approaches the set of easily accessible and spontaneously activated exemplars of the two categories. Based on the above described criteria, the categories carnivores and herbivores were selected.

\section{Participants}

One hundred and seventy-three students from the University of Leuven participated in this experiment. Ten graduate students who participated voluntarily completed an exemplar generation task. One hundred and one first-year students, participating for course credit, completed a feature generation task. Ten paid first-year students filled out a feature applicability matrix. Twenty research graduates and students took part in a naming study and 32 students from different faculties participated in a categorization task voluntarily.

\section{Procedure}

Stimulus generation task. To approximate the set of possibly activated exemplars of both categories, a stimulus generation task was conducted. In this task, the participants were asked to generate 10 animals for both the categories carnivores and herbivores. Every animal that was mentioned by at least two (of the 10) participants was included in the category exemplar set. Applying this criterion, 73 well-known animals were retained, of which 35 were carnivores and 38 herbivores. Next, 28 animals were added that were presumably unknown to a population of European students. A list of the 73 wellknown and 28 novel carnivores and herbivores is given in Appendix A. Fifteen of the novel animals were carnivores; the remaining 13 were herbivores.

Feature generation task. Next, a feature generation task was conducted. Every participant in this task was shown 10 of the well-known animal stimuli and was asked to list 10 features for each of them. This task took approximately $45 \mathrm{~min}$. Presentation of the stimuli over the participants was random with the restriction that every well-known animal was presented to 10 different people. Features that were generated at least 40 times (tallied over all stimuli and participants) were included in the feature list. This resulted in a list of 39 different features. Finally, for all 101 stimuli, high quality photographs were selected that did not display any indication regarding the animal's dietary habits.

Feature applicability task. In the feature applicability task, each participant was presented with an Excel-file. Each animal picture, together with the 39 features, was presented on a different sheet. Participants were asked to indicate whether the features were applicable to the respective animals ( 0 or 1 for no and yes, respectively). They were instructed to make as good a guess as possible when in doubt. Every participant rated all 101 animals. The task took approximately two hours.

Naming task. In the naming task, the photographs of the 28 unknown animals were presented on a screen one by one (in two different random orders). The partic- 
ipants had to answer whether they knew the pictured animal. If they thought they knew it, they were asked to name the animal at the most specific level of abstraction that they were able to. Their answers were recorded by the experimenter.

Categorization task. In the final task, stimuli were presented in the middle of a computer screen, one pictured animal at a time. Participants were instructed to indicate whether the animal was a carnivore or a herbivore by pressing the left button or the right button, respectively. After responding, participants were shown an "o.k." message and were instructed to press the space bar to go to the next picture. Participants were allowed to take a short break when their concentration dropped. After pressing, the next picture was presented. All 101 animals were presented randomly without time limit. Participants were instructed to categorize the stimuli as well as possible, and to always give an answer. The task took approximately $10 \mathrm{~min}$. The well-known carnivores and herbivores were categorized as herbivores in $94 \%$ and $6 \%$ of the cases, respectively. For the unknown animals, $43 \%$ of carnivore classifications were made, with proportions for individual animals varying from 0 to $100 \%$. Details about the observed proportions are given in Appendix A together with the animal names.

\section{MDS analyses}

A matrix of 101 animals $\times 39$ features was obtained by adding, for each cell, the responses of the 10 participants in the feature applicability task. Hence, a number between 0 and 10 appeared in every cell, indicating the extent to which the participant group as a whole considered a feature applicable to a certain animal. Every animal had a feature applicability vector of 39 features. One may question the validity of this similarity measure because the participants were forced to make decisions about features of completely unknown animals. Therefore, the interrater consistency of each of the feature vectors of every stimulus was estimated using the split-half technique. Very high values were obtained, ranging from .82 to, .99 , with only 8 of the 101 estimates below .90 .

By correlating these vectors for every possible pair of animals, a similarity matrix was obtained. As in Smits et al. (2002), a value of 1 was added to all correlations to ensure that all values were positive (as required by the multidimensional scaling algorithm that was used). Consequently, the matrix was analyzed using the MDS procedure in the statistical package SAS (SAS Institute Inc, 2003 user manual), a procedure that shares many characteristics with the ALSCAL procedure (Takane et al., 1977). The Stress was .30, .17, .11, .08, .06, .05 and .04 in 1 to 7 dimensions, respectively. Based on this stress profile, we decided to investigate solutions in dimensionalities 2 and 3 .
Fitting the K-means Varying Abstraction Framework

The modeling procedure was identical to the procedure outlined in Study 1. First, $K$-means clustering was applied to the categories herbivores and carnivores separately, for both the two-dimensional and the threedimensional solution. The analyses resulted in 38 different partitions for herbivores and 35 different partitions for carnivores, defining $38 \times 35=1330$ models to be tested for both MDS-solutions. Note that, whereas the MDS solution was based on all 101 animals, the clustering only involved the well-known animals. Because it is possible that the similarity among the to-be-clustered items are affected by the presence of the novel items, we re-analyzed the input similarities, excluding all novel stimuli, with MDS, i.e., the pairwise similarity matrix of only the 79 well-known stimuli. The resulting representation was very similar to the representation of these same 79 stimuli within the joint MDS analysis based on all 101 stimuli. These results reassured us that applying the clustering on the coordinates of the joint MDS solution did not affect the final clusters.

All models were fitted to the categorization data of the 28 unknown animals using maximum likelihood estimation. The values of the parameters $r$ and $q$ were again set to either 1 or 2 , resulting in 4 possible combinations.

\section{Results and discussion}

The results of the naming task clearly showed that the 28 animals that were selected to be unknown were indeed unfamiliar to the participant population. Of all $(20 \times 28$ $=$ ) 560 responses, $65.7 \%$ were 'don't know' responses. Only seven responses $(1.3 \%)$ were correct at the species level. (One stimulus was named correctly by four participants, one stimulus was named correctly by two participants, and one other stimulus was correctly named by one single participant.) Eleven answers $(2.0 \%)$ were wrong at the species level but correct at the family level (e.g., canines). Very general, but correct, answers, defined at the order level (e.g., rodents), were given in $20.6 \%$ of the cases. All remaining $10.4 \%$ of the responses were wrong. In the majority of these answers (48 out of 58 responses), the incorrectly named animal belonged to a different order as the target animal. Hence, it is a safe assumption that the unknown animals were truly unknown.

We will discuss only the three-dimensional solution with $r=2$ (Euclidean distance) and $q=1$ (exponential decay), as this again resulted in much better fit values. The models were fit with 4 free parameters, as discussed in Study 1. Figs. 3a and $b$ give an overview of the fit values for all 1330 investigated models. Table 3 gives an overview of the fit values and parameter estimates of the Single-Prototype, the Full-Exemplar and the best fitting pseudo-exemplar model.

As in Study 1, the Full-Exemplar Model (i.e., the GCM), with a $-2 \ln (L)$ value of 177.20 , performed better 


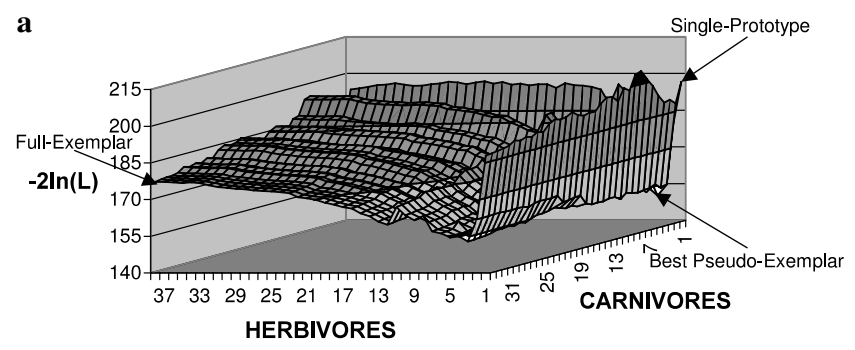

$\square 140-155 \square 155-170 \square 170-185 \square 185-200 \mathbf{\square} 200-215$

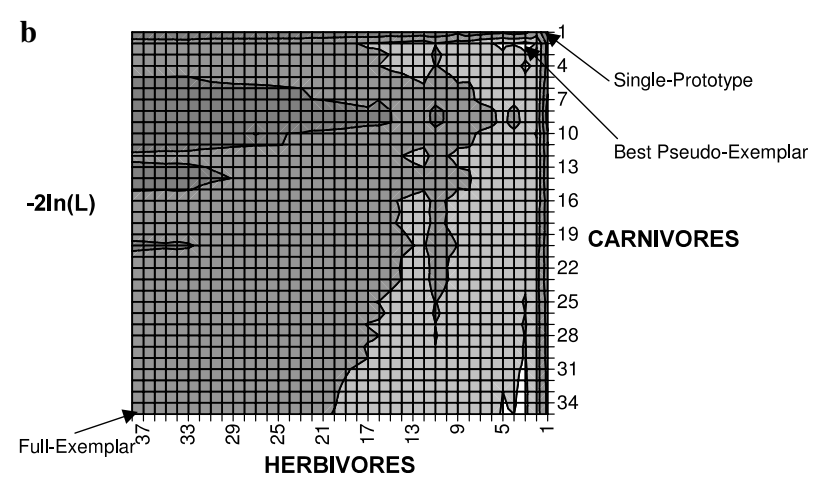

Fig. 3. $-2 \ln (L)$-values for all models for the concepts herbivores and carnivores as seen on a surface plot. The number of clusters for each category is indicated on the axis, (a) gives a view from the side, (b) from the top. Orientations are identical. The optimal, SinglePrototype, and Full-Exemplar model are indicated.

than the Single-Prototype Model which had a fit value of 196.62. Once again, total abstraction was much less plausible than full detail as a representational basis to explain the categorization decisions. However, there were again a vast number of intermediate pseudo-exemplar models with better fit values than the Full-Exemplar and SinglePrototype Models. The best performing pseudo-exemplar model was one with a very high level of abstraction, based on only 3 clusters for herbivores and 2 clusters for carnivores. It had a $-2 \ln (L)$ value of 150.72 . The differences between the Single-Prototype Model and the best fitting model, and the Full-Exemplar Model and the best fitting model $(177.20-150.72=26.48$ and $196.62-150.72=$ 45.90 , respectively) are large enough to reject both the Single-Prototype Model and the Full-Exemplar Model as the best-fitting models.

Just like in Study 1, an asymmetric pattern could be detected in the number of activated representations in both categories. As indicated above, the best fitting model was based on two carnivore clusters and three herbivore clusters. In the second best model, a similar, but even stronger asymmetry was found with two carnivore and five herbivore clusters.

Table 4 shows the cluster assignment for the optimal 3 herbivore and 2 carnivore clusters. The clusters are readily interpretable. The category carnivores fell apart in land-dwelling animals on the one hand, and seaand air-dwelling animals on the other hand. Herbivores consisted of a cluster for small to average sized mammals and/or marsupials, one for very large mammals, and a small cluster of birds together with reptiles.

To sum up, we replicated the results from Study 1 with different categories and with a different feature generation task. Though the Full-Exemplar Model outperformed the Single-Prototype Model, there are better models, predicting categorization on the basis of a limited number of pseudo-exemplars. We also achieved our second goal to obtain a more attractive clustering

Table 3

Fit value $(-2 \ln (L))$, explained variance $\left(R^{2}\right)$, sensitivity $(\mathrm{c})$, bias $\left(\beta_{\mathrm{A}}\right)$ for the category carnivores, and dimension weights for the SinglePrototype, Full-Exemplar, and best fitting pseudo-exemplar model in Study 2

\begin{tabular}{lccccccc}
\hline Model & $-2 \ln (L)$ & $R^{2}$ & $c$ & $\beta_{\mathrm{A}}$ & $w_{1}$ & $w_{2}$ \\
\hline Single-prototype & 196.62 & .76 & 2.043 & 0.598 & 0.321 & 0.470 & 0.209 \\
Full-exemplar & 177.20 & .80 & 5.865 & 0.546 & 0.483 & 0.159 & 0.358 \\
Best pseudo-exemplar & 150.72 & .88 & 2.080 & 0.709 & 0.911 & 0.055 & 0.034 \\
\hline
\end{tabular}


Table 4

Clusters produced for the concepts herbivores and carnivores in the best fitting pseudo-exemplar model

$\begin{array}{ll}\text { Carnivores } & \\ \text { Cluster } 1 & \text { Eagle, dolphin, vulture, shark, hawk, crocodile, killer whale, piranha, python, heron, snake, pike, owl, falcon, whale, sea lion } \\ \text { Cluster } 2 & \text { Bear, cheetah, dog, hyena, polar bear, jaguar, jackal, cat, lion, leopard, lynx, otter, panther, puma, tiger, bat, fox, raccoon, wolf }\end{array}$

\section{Herbivores}

Cluster 1 Monkey, guinea pig, squirrel, goat, hare, hamster, kangaroo, koala, rabbit, sloth, mouse, rat, sheep, pig

Cluster 2 Duck, chicken, turtle

Cluster 3 Antelope, bison, buffalo, dromedary, moose, donkey, giraffe, wildebeest, gorilla, deer, camel, cow, lama, rhinoceros, hippo, okapi, elephant, horse, panda, roe, zebra

solution: the subsets underlying these pseudo-exemplars are clearly interpretable for both categories. This suggests that expanding the set of features as a basis for the similarity matrix, by letting features be generated at the level of the actual concept members, was indeed effective in avoiding semantically implausible clusters.

\section{General discussion}

In this paper, we applied a model that is based on the idea of varying abstraction as a basis for categorization. More specifically, we used the Varying Abstraction Framework, a family of models that ranges from complete abstraction (i.e., a single summary representation) to no abstraction at all (i.e., every exemplar is represented in memory and activated in categorization decisions) (Vanpaemel \& Storms, 2006; Vanpaemel et al., 2005). As natural speakers, we presumably do not activate all possible memorized members of a concept, even though we have no problem recalling the individual members. Similarly, a single summary representation seems to fall short in explaining the relative accuracy with which we handle larger, more heterogeneous concepts. The Varying Abstraction Framework allows intermediate levels of abstraction, in which some exemplars are merged together in a single representation and other exemplars are kept apart in separate representations. These intermediate levels of abstraction can, but do not have to correspond to lexicalized categories. By comparing the fit of models that vary in abstraction, this approach is suited to track down the level of abstraction that best accounts for categorization decisions.

Fitting all possible pseudo-exemplar models of the Varying Abstraction Framework when categories are large, as was the case in our two studies, is intractable. Furthermore, in the case of well-known categories, it seems implausible that the partitions people use are not guided by unsupervised processes on the (similarity) structure of stimuli involved. Indeed, it has been argued that, in the case of large natural language categories, people learn through ostensive (from "ostendere", to show) learning (Schyns, 1991), meaning that there is only limited supervised feedback, and there is a considerably com- plex structure of the stimulus domain. In other words, unsupervised processes seem to constrain the partitions people are likely to explore (Schyns, 1991; Pothos \& Chater, 2002). Therefore, we introduced a special version of the Varying Abstraction Framework in which only one partition is considered at every level of abstraction. The $K$-means Varying Abstraction Framework is particularly suited to analyze categorization data in superordinate level natural concepts for at least three reasons. First, most natural language concepts are embedded in a hierarchical structure, which raises a number of problems for the traditional prototype and exemplar models. Second, natural categories tend to be quite large. Any acceptable model therefore requires a plausible unsupervised organizational principle if assumptions about human computational capacity are to be kept plausible. Finally, most of the concepts studied in natural language are relatively well-known. As the $K$-means Varying Abstraction Framework is intended to describe memory representations at learning asymptote, the different levels (in terms of number of clusters) of $K$ means clustering provide a natural insight into the abstraction level of the memory representations that get activated, rather than making assumptions about a (very long and past) learning process.

As noted above, the $K$-means Varying Abstraction Framework presented in this paper does not specify when the unsupervised learning process takes place. In terms of the applications presented in this paper, our approach does not need to specify whether the unsupervised clustering occurred in the past, when the participants learned the well-known exemplars of the studied categories, or during the experiment, when they were asked to categorize the novel stimuli.

The two applications of categorization in superordinate level natural concepts presented in this paper clearly showed that models based on intermediate levels of abstraction outperformed both the traditional SinglePrototype Model and a Full-Exemplar Model with exemplars defined at the basic level (Rosch et al., 1976). To our knowledge, this is the first demonstration that models based on intermediate abstration outperform the two traditional approaches in explaining categorization decisions for natural categories. 
Advocates of the exemplar view might argue, though, that the exemplar model tested in our studies cannot be considered as an implementation of their ideas, because the assumed basic level representations still abstract over a wide range of encountered exemplars (Nosofsky, 1988; Barsalou et al., 1998). Although this is undoubtedly true, we would like to raise three points that weaken this argument. First, because of the extremely large number of encountered exemplars of most superordinate natural language concepts, it is intuitively hard to assume that memory traces of all encountered exemplars are stored and activated in category-related decisions. Second, most individual exemplars of basic level categories (like bikes, rhubarb, foxes, etc.) are highly similar. For many of these basic level categories (e.g., robin, trout, etc.) one can doubt whether people are able at all to distinguish between different category exemplars. Third, because the learning history of participants is so utterly different and untraceable, a version of the exemplar view that assumes no abstraction at all seems completely unfalsifiable. Therefore, until someone comes up with a way to rigorously test this hypothesis, this theoretical idea is not constructive in our understanding of human memory and its role in categorization.

The two studies also showed that there may be an asymmetry in how detailed the activated representation is of two rivaling categories in a categorization decision. Not only the very best fitting model, but also other well fitting competitor models in Study 1 were based on more clusters for fruits than for vegetables. Likewise, the best fitting models in Study 2 were based on more clusters for herbivores than for carnivores. These findings show how the Varying Abstraction Framework is capable of detecting regularities in the representation details of semantic concepts.

One may question how our results relate to the main findings from the artificial category learning tradition and from the semantic concept tradition. In the majority of the artificial category learning studies (e.g., Medin \& Schaffer, 1978; Medin et al., 1984; Nosofsky, 1992), different versions of the exemplar model have been shown to outperform the Single-Prototype Model. However, recently Smith and Minda (2000); Smith and Minda (2002) showed that this conclusion may need some nuances and that under certain conditions, abstraction processes take place. We think that further investigation may benefit from including systematic checking of the level of abstraction that best accounts for categorization decisions under certain circumstances and that abstraction levels in between those corresponding to the Single Prototype and Full-Exemplar Models can be useful in settling the debate (see Vanpaemel et al., 2006, for an example).

Given the large number of models that are generated and compared in the $K$-means Varying Abstraction Framework, one may ask how well the approach is able to retrieve the correct model and how sensitive it is to overfitting the data. The answer to this question obviously depends on the structure of the categorization task and the size of the data set. In a recent simulation study, Vanpaemel and Storms (2006) focused on the ability of the Varying Abstraction Framework to recover the best fitting instantiation of the model family. In this study, a data set from Nosofsky, Shin, and Clark (1989) was first analyzed with all the different models described by the varying abstraction approach. In this study, the categories had exemplar sets of size 3 and 4, respectively, resulting in 75 different models (including the FullExemplar and the Single Prototype models) to fit to the data. One intermediate abstraction model was found to outperform all others (including the Single Prototype and the Full-Exemplar Model) in predicting the empirical data. Next, a simulation study was set up in which 100 data sets, equal in size to the empirical data set, were generated according to each of the 75 models of the Varying Abstraction Framework. The parameter values used to generate the simulated data were the optimal parameter values obtained when analyzing the empirical data of the corresponding models. All simulated data sets were then analyzed with all 75 models of the Varying Abstraction Model family (i.e., $75 \times 75 \times 100=562.500$ analyses). The results showed that the recovery of the true underlying model (i.e., the model on which the simulated data were based) was very good. In $98 \%$ of the analyzed data sets, the generating model provided the best fit, showing that, even when challenged with 74 competitors, the correct model recovers its own data best.

\section{Relation with other intermediate abstraction models}

The $K$-means Varying Abstraction Framework is not the first to entertain some formalization of varying abstraction. Examples include Anderson's (1991) Rational Model; Schyns' (1991) Modular Network Model, and Love et al.'s (2004) SUSTAIN. The main difference between these models and our own lies in the position our model takes concerning learning history. The aforementioned models take on formal assumptions about a learning history and try to simulate this history. They are guided by an iterative process that determines final partitioning and the aim is to determine whether the learning assumptions of any particular model can better explain the categorization data than those of a competing model. In the Varying Abstraction Framework, no assumptions about learning history are made, save that learning is ostensive for natural speakers. It is therefore primarily intended to uncover the relative plausibility of memory representations associated with category labels at a late stage of learning.

Another model framework with varying abstraction but without learning process assumptions is the Mixture Model Framework of categorization, proposed by Rosseel (2002). It represents a category by a mixture 
of $K$ distributions, with $K$ varying from 1 to $N$. The clusters of the $K$-means Varying Abstraction Framework can in principle be formalized as a mixture of distributions. One of the specific models of the Mixture framework, called the Reduced Exemplar Model, is in fact highly similar and under certain conditions identical to the $K$-means variant of the Varying Abstraction Model. The latter model was intended as both a practical and psychologically appealing simplification of the general Varying Abstraction Framework, suited for applications to natural language concepts.

To summarize, unlike several earlier models that rely on partial abstraction, our framework is specifically aimed at uncovering memory representations of existing categories, in a context of classification of novel items in existing categories. We argue that the study of natural language concepts may benefit from such a systematic comparison of different latent abstraction levels, an approach that has been largely neglected in this context due to the predominant approach of comparing models that differ in their assumptions about learning history, rather than their assumptions about memory representations.

The $K$-means Varying Abstraction Framework is the first approach to our knowledge that tries to uncover the relative plausibility of memory representations associated to category labels, in a context where there is little a priori knowledge about the dimensions or features that people attend to. It starts with defining a similarity structure that best captures the similarity of items, and then assumes related levels of gradually increasing accuracy based on related steps in an unsupervised process. Research along these lines was initiated by Heit and Barsalou (1996) and De Wilde et al. (2003), whose results cast doubt on the generality of the prototype and exemplar views on semantic concepts that have long dominated the research (e.g., Hampton, 1979; Rosch \& Mervis, 1975; Nosofsky, 1992; Smith \& Minda, 2000, 2002). We believe that the $K$-means Varying Abstraction Framework used in this paper may further our understanding of semantic concept representation.

\section{Appendix A}

1a. Names and observed proportion fruit decisions of the 35 fruits used in Study 1

Meddler

Blackberry

Raspberry

Peanuts

Chestnut

Nectarine

Pomegranate

Fig

Cactus fruit

Papaya
Litchi

Pineapple

1.00

Coconut

1.00

Kiwi

1.00

Ground-nut

Mango

1.00

0.90

Apple

0.90

Avocado

1.00

Star fruit

0.20

0.10

Pear

Orange

Lemon

Melon

Passion fruit

Walnut

Grapes

Tangerine

Plum

Grapefruit

Banana

Date

Lime

Rhubarb

Cherries

Strawberry

1.00

1.00

1.00

0.87

0.97

0.97

1.00

1.00

1.00

1.00

1.00

1.00

0.97

0.33

1.00

0.93

1b. Names and observed proportion

fruit decisions of the 44 vegetables used in study 1

Lentils

0.57

Chick peas

0.80

Princess broad bean

0.00

Brussels sprouts

0.00

Peppers

0.00

Egg plant

0.17

Artichoke

0.07

Chicory

0.00

Cucumber

Red cabbage

0.00

0.00

Broccoli

0.00

Cauliflower

0.00

Celery

Window-nasty

0.00

White cabbage

0.10

0.00

Parsley

Pipe onion

Leek

0.00

0.00

Carrots

Garlic

Tomato

0.00

0.00

0.00

0.10

Turnip

0.00

Lettuce

0.00

Radish

Mushroom

0.03

0.00

Celeriac

0.00

Pumpkin

Fennel

Zucchini

Paprika

Corn

Haricots

0.30

0.00

0.00

0.03

0.00

0.00

0.03

Beans

0.00 


\begin{tabular}{|c|c|c|c|}
\hline Potatoes & .00 & Leopard & 1.00 \\
\hline Chives & .00 & Lynx & 1.00 \\
\hline Spinach & .00 & Killer whale & 0.94 \\
\hline Asparagus & .00 & Otter & 0.91 \\
\hline Peas & .13 & Panther & 1.00 \\
\hline Gherkin & .03 & Piranha & 0.88 \\
\hline Endives & .00 & Puma & 1.00 \\
\hline Chervil & .00 & Python & 1.00 \\
\hline Sea weed & .03 & Heron & 0.94 \\
\hline \multirow[t]{2}{*}{ Black salsify } & .00 & Snake & 0.91 \\
\hline & & Pike & 0.63 \\
\hline 1c. Names and observed proportions of fruit & & Tiger & 1.00 \\
\hline decisions of the 30 novel foods used in study 1 & & Owl & 0.97 \\
\hline Lemon grass & .00 & Falcon & 1.00 \\
\hline Mangosteen & .70 & Bat & 0.88 \\
\hline Kiwano & .90 & Fox & 1.00 \\
\hline Bitter lemon & .05 & Whale & 0.63 \\
\hline Tomarillo & .85 & Raccoon & 0.59 \\
\hline Okra & .05 & Wolf & 1.00 \\
\hline Turmeric & .00 & Sea lion & 0.94 \\
\hline Thai eggplant & .10 & & \\
\hline Guaga & .00 & 2b. Names and observed proportion of carnivore & \\
\hline Bergamot & .70 & decisions of the 38 herbivores used in Study 2 & \\
\hline Lilac & .10 & Monkey & 0.03 \\
\hline Topinanbour & .00 & Antelope & 0.06 \\
\hline Rambutan & .95 & Bison & 0.06 \\
\hline Edos & .95 & Buffalo & 0.09 \\
\hline Kumquat & .65 & Guinea pig & 0.03 \\
\hline Patisson & .55 & Dromedary & 0.03 \\
\hline Kan-toon & .20 & Squirrel & 0.00 \\
\hline Noix-de-cola & .40 & Duck & 0.13 \\
\hline Big gourd & .35 & Mmoose & 0.09 \\
\hline Pitahaya & .65 & Donkey & 0.00 \\
\hline Patat douce & .00 & Goat & 0.00 \\
\hline Tiny korella & .70 & Giraffe & 0.00 \\
\hline Jujube & .90 & Wildebeest & 0.09 \\
\hline Cherimoya & .65 & Gorilla & 0.09 \\
\hline Ripe tamarind & .40 & Hare & 0.00 \\
\hline Young peper & .85 & Hamster & 0.09 \\
\hline Banana blossom & .10 & Deer & 0.06 \\
\hline Taro & .30 & Camel & 0.03 \\
\hline Cayotte & .50 & Kangaroo & 0.00 \\
\hline \multirow[t]{2}{*}{ Safon } & .40 & Chicken & 0.16 \\
\hline & & Koala & 0.00 \\
\hline \multirow{2}{*}{\multicolumn{2}{|c|}{$\begin{array}{l}\text { 2a. Names and observed proportion of carnivore decisions of the } \\
35 \text { carnivores used in study } 2\end{array}$}} & Cow & 0.03 \\
\hline & & Rabbit & 0.00 \\
\hline Eagle & .00 & Lama & 0.00 \\
\hline Bear & .00 & Sloth & 0.00 \\
\hline Cheetah & .00 & Mouse & 0.19 \\
\hline Dolphin & .84 & Rhinoceros & 0.03 \\
\hline Vulture & .00 & Hippo & 0.31 \\
\hline Shark & .97 & Okapi & 0.06 \\
\hline Hawk & .00 & Elephant & 0.03 \\
\hline Dog & .00 & Horse & 0.03 \\
\hline Hyena & .00 & Panda & 0.06 \\
\hline Polar bear & .00 & Rat & 0.19 \\
\hline Jaguar & .00 & Roe & 0.03 \\
\hline Jackal & .00 & Sheep & 0.03 \\
\hline Cat & .00 & Turtle & 0.09 \\
\hline Crocodile & .00 & Pig & 0.28 \\
\hline Lion & .00 & Zebra & 0.03 \\
\hline
\end{tabular}


2c. Latin names and observed proportions of carnivore decisions of the 28 novel animals used in Study 2

Orychterapus afer

Apteryx australis mantelli

Arctictis binturong

Budorcas taxicolor

Cacajao rubicundus

Capricornis sumatraensis argyrachaetes

Chlamyphorus truncatus

Colobus caudatus

Cynictis penicillata

Cynocephalus volans

Dendrolagus matschiei

Dolichotis patagonum

Draco volans linnaeus

Genetta

Hyemoschus aquaticus

Indri

Lama vicugna

Lasiorhinus latifrons

Lutreolina crassicaudata

Macroclemys temminckii

Metachirus opossum

Otocyon megalotis

Perodicticus potto

Phalanger maculatus

Salamandra japonicus

Salamandra

Setonix brachyurus

Speothos venaticus

Carnivore $\quad 0.72$
Carnivore $\quad 0.44$

Carnivore $\quad 0.56$

Herbivore $\quad 0.06$

Herbivore $\quad 0.16$

Herbivore $\quad 0.16$

Carnivore 0.59

Herbivore 0.13

Carnivore 0.56

Herbivore 0.38

Herbivore $\quad 0.25$

Herbivore $\quad 0.00$

Carnivore 0.78

Carnivore $\quad 0.91$

Herbivore 0.31

Carnivore $\quad 0.09$

Herbivore $\quad 0.06$

Herbivore 0.19

Herbivore $\quad 0.78$

Carnivore $\quad 0.44$

Herbivore $\quad 0.56$

Carnivore $\quad 1.00$

Carnivore $\quad 0.25$

Carnivore $\quad 0.16$

Carnivore $\quad 0.72$

Carnivore $\quad 0.72$

Herbivore $\quad 0.16$

Carnivore $\quad 0.81$

\section{References}

Anderson, J. R. (1991). The adaptive nature of human categorization. Psychological Review, 98, 409-429.

Barsalou, L. W. (1990). On the indistinguishability of exemplar memory and abstraction in memory representation. In T. K. Srull \& R. S. Wyer (Eds.). Advances in social cognition (pp. 61-88). Hillsdale, NJ: Erlbaum.

Barsalou, L. W., Huttenlocher, J., \& Lamberts, K. (1998). Basing categorization on individuals and events. Cognitive Psychology, 36, 203-272.

De Wilde, E., Vanoverberghe, V., Storms, G., \& De Boeck, P. (2003). The instantiation Principle Re-evaluated. Memory, 11, 533-548.

Elvevåg, B., Storms, G., Heit, E., \& Goldberg, T. (2005). Category content and structure in schizophrenia: An evaluation using the instantiation principle. Neuropsychology, 19, 371-380.

Estes, W. K. (1994). Classification and Cognition. New York/ Oxford: Oxford University Press.

Hampton, J. A. (1979). Polymorphous concepts in semantic memory. Journal of Verbal Learning and Verbal Behavior, $18,441-461$.

Hampton, J. A. (1993). Prototype models of concept representation. In I. VanMechelen, J. Hampton, R. S. Michalski, \& P. Theuns (Eds.), Categories and concepts: Theoretical views and inductive data analysis (pp. 67-96). London, England: Academic Press.
Hastie, T., Tibshirani, R., \& Friedman, J. (2001). The elements of statistical learning: Data mining, inference and prediction. New York: Springer-Verlag.

Heit, E., \& Barsalou, L. W. (1996). The instatiation principle in natural language categories. Memory, 4, 413-451.

Herz, J., Krogh, A., \& Palmer, R. G. (1991). Introduction to the theory of neural computation. Redwood City, CA: AddisonWesley.

Kass, R. E., \& Raftery, A. E. (1995). Bayes factors. Journal of the American Statistical Association, 90, 773-795.

Komatsu, L. K. (1992). Recent views of conceptual structure. Psychological Bulletin, 3, 500-526.

Lakoff, G. (1987). Woman, Fire, and Dangerous Things; What Categories Reveal about the Mind. Chicago and London: University of Chicago Press.

Lamberts, K. (2000). Information-accumulation theory of speeded categorization. Psychological Review, 107, 227-260.

Lee, M. D. (2001). Determining the dimensionality of multidimensional scaling models for cognitive modeling. Journal of Mathematical Psychology, 45, 149-166.

Love, B. C., Medin, D. L., \& Gureckis, T. M. (2004). SUSTAIN: A network model of category learning. Psychological Review, 11, 309-332.

Malt, B. C., \& Smith, E. E. (1984). Correlated properties in natural categories. Journal of Verbal Learning and Verbal Behaviour, 23, 250-269.

Markman, A. B., \& Ross, B. H. (2003). Category use and category learning. Psychological Bulletin, 129, 592-613.

McRae, K., Cree, G. S., Westmacott, R., \& de Sa, V. R. (1999). Further evidence for feature correlations in semantic memory. Canadian Journal of Experimental Psychology: Special Issue on Models of Word Recognition, 53, 360-373.

Medin, D. L., Altom, M. W., \& Murphy, T. D. (1984). Given versus induced category representations: use of prototype and exemplar information in classification. Journal of Experimental Psychology: Learning, Memory, and Cognition, 3, 333-352.

Medin, D. L., \& Coley, J. D. (1998). Concepts and categorization. In J. Hochberg \& J. E. Cutting (Eds.), Handbook of perception and cognition: Perception and cognition at century's end (2nd ed., pp. 403-440). San Diego: Academic Press.

Medin, D. L., \& Schaffer, M. M. (1978). Context theory of classification learning. Psychological Review, 85, 207-238.

Murphy, G. L. (2002). The big book of concepts. Cambridge, MA: MIT Press.

Murphy, G. L. (2003). Ecological validity and the study of concepts. In B. H. Ross (Ed.). The psychology of learning and motivation (Vol. 43, pp. 1-41). San Diego: Academic Press.

Nosofsky, R. M. (1984). Choice, similarity, and the context model of classification. Journal of Experimental Psychology: Learning, Memory, and Cognition, 10, 104-114.

Nosofsky, R. M. (1986). Attention, similarity, and the identification-categorization relationship. Journal of Experimental Psychology: General, 115, 39-57.

Nosofsky, R. M. (1988). Similarity, frequency, and category representations. Journal of Experimental Psychology: Learning, Memory, and Cognition, 14, 54-65.

Nosofsky, R. M. (1992). Exemplars, prototypes and similarity rules. In A. F. Healy, S. M. Kosslyn, \& R. M. Shiffrin (Eds.), From learning theory to connectionist theory: Essays 
in honour of William K. Estes, Vol. 1. Hillsdale, NJ: Lawrence Erlbaum.

Nosofsky, R. M., \& Johansen, M. K. (2000). Exemplar-based accounts of "multiple-system" phenomena in perceptual categorization. Psychonomic Bulletin and Review, 7, 375-402.

Nosofsky, R. M., Shin, H. J., \& Clark, S. E. (1989). Rules and exemplars in categorization, identification, and recognition. Journal of Experimental Psychology: Learning, Memory and Cognition, 15, 282-304.

Pothos, E. M., \& Chater, N. (2002). A simplicity principle in unsupervised human categorization. Cognitive Science, 26, 303-343.

Reed, S. K. (1972). Pattern recognition and categorization. Cognitive Psychology, 3, 382-407.

Rips, L. J. (1989). Similarity, typicality, and categorization. In A. Orthony \& S. Vosniadou (Eds.), Similarity and analogical reasoning (pp. 21-59). New York, NY: Cambridge University Press.

Rogers, T. T., \& McClelland, J. L. (2004). Semantic Cognition: A Parallel Distributed Processing Approach. Cambridge, MA: MIT Press.

Rosch, E. (1975). Cognitive representation of semantic categories. Journal of Experimental Psychology, 104, 573-605.

Rosch, E. (1978). Principles of categorisation. In E. Rosch \& B. Lloyd (Eds.), Cognition and Categorisation (pp. 27-48). Hillsdale, N.J: Erlbaum.

Rosch, E., \& Mervis, C. B. (1975). Family resemblances: Studies in the internal structure of categories. Cognitive Psychology, 7, 573-605.

Rosch, E., Mervis, C., Gray, W., Johnson, D., \& Boyes-Braem, P. (1976). Basic Objects in Natural Categories. Cognitive Psychology, 8, 382-439.

Rosseel, Y. (2002). Mixture models of categorization. Journal of Mathematical Psychology, 46, 178-210.

Ross, B. H., \& Murphy, G. L. (1999). Food for thought: Crossclassification and category organization in a complex realworld domain. Cognitive Psychology, 38, 495-553.

Rumelhart, D. E., \& Zipser, D. (1985). Feature discovery by competitive learning. Cognitive Science, 9, 75-112.

SAS Institute Inc. (2003). SAS OnlineDoc 9.1. [Computer manual]. Retrieved June 3, 2004, from http://support.sas.com/91doc/docMainpage.jsp.

Schyns, P. (1991). A modular neural network model of concept acquisition. Cognitive Science, 15, 461-508.

Smith, E., \& Medin, D. (1981). Categories and Concepts. Cambridge, Mass: Harvard University Press.

Smith, J. D., \& Minda, J. P. (2000). Thirty categorization results in search of a model. Journal of Experimental Psychology: Learning, Memory and Cognition, 26, 3-27.
Smith, J. D., \& Minda, J. P. (2002). Distinguishing prototypebased and exemplar-based processes in category learning. Journal of Experimental Psychology: Learning, Memory, and Cognition, 28, 800-811.

Smits, T., Storms, G., Rosseel, Y., \& De Boeck, P. (2002). Fruits and vegetables categorized: An application of the generalized context model. Psychonomic Bulletin and Review, 9, 836-844.

Storms, G. (2004). Exemplar models in the study of natural language concepts. In B. H. Ross (Ed.), The Psychology of Learning and Motivation. New York: Academic Press.

Storms, G., De Boeck, P., \& Ruts, W. (2000). Prototype and exemplar-based information in natural language categories. Journal of Memory and Language, 42, 51-73.

Storms, G., De Boeck, P., \& Ruts, W. (2001). Categorization of unknown stimuli in well- known natural language concepts: a case study. Psychonomic Bulletin and Review, 8, 377-384

Storms, G., De Boeck, P., Van Mechelen, I., \& Ruts, W. (1996). The dominance effect in concept conjunctions: Generality and interaction aspects. Journal of Experimental Psychology: Learning, Memory and Cognition, 22, 1-15.

Takane, Y., Young, F. W., \& De Leeuw, J. (1977). Nonmetric individual differences multidimensional scaling: An alternating least squares method with optimal scaling features. Psychometrika, 42, 7-67.

Vanpaemel, W., \& Storms, G. (2006). A varying abstraction framework for categorization. Manuscript submitted for publication.

Vanpaemel, W., Storms, G., \& Ons, B. (2005). A varying abstraction model for categorization. In B. Bara, L. Barsalou, \& M. Bucciarelli (Eds.), Proceedings of the 27th annual conference of the Cognitive Science Society (pp. 2277-2282). Mahwah, NJ: Lawrence Erlbaum.

Vanpaemel, W., Pattyn, S. \& Storms, G. (2006). Yet another look at thirty categorization results. In R. Sun \& N. Miyake (Eds.), Proceedings of the 28th Annual Conference of the Cognitive Science Society (pp. 2299-2304). Mahwah, NJ: Lawrence Erlbaum.

Verbeemen, T., Vanoverberghe, V., Storms, G., \& Ruts, W. (2001). The role of contrast categories in natural language concepts. Journal of Memory and Language, 44, 618-643.

Verguts, T., Ameel, E., \& Storms, G. (2004). Measures of similarity in models of categorization. Memory \& Cognition, 32, 379-389.

Verguts, T., Storms, G., \& Tuerlinckx, F. (2003). Decision bound theory and the influence of familiarity. Psychonomic Bulletin \& Review, 10, 141-148. 